\title{
RBIS - An Environmental Information System for Integrated Landscape Management
}

\author{
Franziska Zander ${ }^{1}$, Sven Kralisch $^{1}$, Carsten Busch $^{2}$, and Wolfgang-Albert Flügel ${ }^{1}$ \\ ${ }^{1}$ Department of Geoinformatics, Hydrology and Modelling, \\ School of Chemical and Earth Sciences, Friedrich-Schiller-University, Jena, Germany \\ ${ }^{2}$ Codematix GmbH, Felsbachstraße 5/7, 07745 Jena, Germany \\ \{Franziska.Zander, Sven.Kralisch, \\ Wolfgang.Albert.Fluegel\} @uni-jena.de, \\ Carsten.Busch@codematix.de
}

\begin{abstract}
In this paper we present the web-based River Basin Information System (RBIS) for data management, analysis and exchange as an integral standalone part of the Integrated Landscape Management System (ILMS). Its architectural layout will be outlined together with the underlying software platform. Selected RBIS modules will be characterized in more detail to emphasize the benefits of integrated data management as a basis of the holistic environmental planning workflow covered by ILMS.
\end{abstract}

Keywords: environmental information systems, integrated landscape management, hydrological modeling.

\section{Introduction}

The need for a detailed understanding of environmental systems and strategies for their adequate management is gaining more and more importance as we face complex problems arising from global climate change, population growth and socio-economic development. In order to create strategies for the sustainable use and management of such systems and to assess the complex interactions of their underlying processes, the integration of data from different sources and disciplines is unavoidable, e.g. from remote sensing, hydrology, biology, soil science or socio-economic sciences. To transfer these data into well-presented and meaningful information for decision makers, they usually have to run through a workflow of different processing steps. This may include data preparation and storage, integrated data analysis by means of methods from various scientific disciplines, the development of future development scenarios and the application of environmental simulation models. Therefore, integrated environmental management systems are needed to address all parts of this workflow as well as a strategy to handle the needed input and produced output data.

\section{Integrated Landscape Management System (ILMS)}

The Integrated Landscape Management System (ILMS) developed at the Department of Geoinformatics, Hydrology and Modelling at the Friedrich Schiller University of 
Jena provides an integrated, modular software platform to address the above mentioned needs and covers different steps of environmental system analysis and planning in a flexible and user-friendly workflow (http://ilms.uni-jena.de). This includes the following integrated but also standalone and mainly open-source platform components:

- ILMSinfo: the River Basin Information System (RBIS) for the management, analysis, visualization and presentation of different types of data.

- ILMSimage: a software for the identification and classification of real-world objects from satellite imagery using methods of object based image analysis [3].

- ILMSgis: a software (GRASS-HRU) for the derivation of modelling entities using a Web Processing Service based on GRASS GIS and following the Hydrological Response Unit (HRU) approach (http://www.geogr.unijena.de/jamswiki /index.php/GRASS-HRU_en) [4].

- ILMSmodel: an environmental modelling framework (Jena Adaptable Modelling System - JAMS) for building, running and analyzing environmental simulation models (http://jams.uni-jena.de) [2].

\section{River Basin Information System (RBIS)}

Providing sophisticated data storage, management and interface functionality, RBIS is one of the core components of ILMS. RBIS is a modular structured web-based information system that offers user-friendly interfaces for the management, analysis, visualization and presentation of different types of data in the context of multidisciplinary environmental assessment and planning. This includes besides metadata (RBISmeta) and datasets of certain content types (e.g. soil (RBISsoil) and vegetation (RBISbio)) also the support for measured or simulated time series data (RBISts), documents (RBISdoc), remote sensing data (RBISrs) and spatial data in raster or vector format (RBISmap). The system was designed to fit the requirements arising from integrated research projects as well as to offer a platform to preserve knowledge beyond their end. Since RBIS is accessible via the web it features a fine grained permission management based on data types (e.g. time series data, soil, geodata, ...) and types of access (e.g. view, edit, delete, ...) to ensure data security and quality control. In addition to the permission management, transparency is ensured by an access logging mechanism which is responsible for recording data manipulations.

The common layout of RBIS follows a standard 3-tier architecture design (figure 1) using the database management system PostgreSQL (http://www.postgresql.org) as storage engine and the extension PostGIS (http://www.postgis.org) to add support for spatial data to PostgreSQL. The whole system is built based on open source software, ensuring a cost-effective deployment and operation. The following sections will shortly explain the underlying software architecture, give an overview of selected RBIS modules and outline some RBIS application examples. 


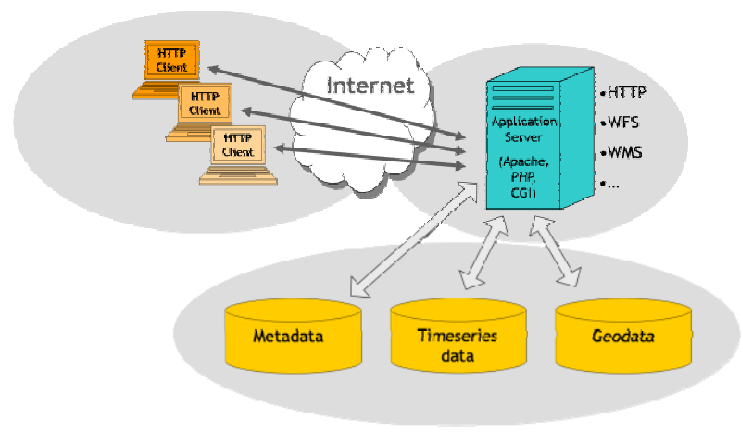

Fig. 1. RBIS technical layout [1]

\subsection{System Architecture}

The development of RBIS was guided by the objective of offering a flexible and modular database and application design that meets emerging demands for new data types and their relationships. This is realized by a data description layer that provides all information needed to manage both, read and writes access to the undelying database as well as the automated creation of web-based user interfaces (figure 2). A XML document describes database relations and attributes that define a certain data type, e.g. a person or a measurement station. The XML document is then evaluated by RBIS in order to create SQL statements and user interfaces needed to perform different actions on these data, e.g. search, browse, edit, add or delete datasets. New data types can easily be added by (i) adopting the database design (e.g. by adding new relations and attributes), and (ii) defining how RBIS shall access these data by means of a new XML document.

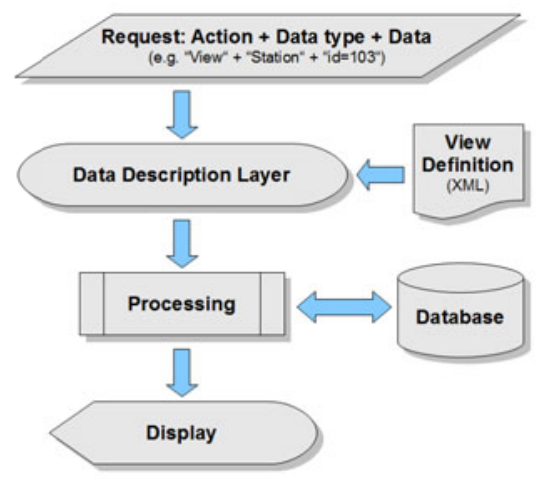

Fig. 2. RBIS information access via data description layer [1]

\subsection{Time Series Data Management (RBISts)}

The module RBISts provides several functions to manage measured or simulated time series data with a special focus on environmental modelling (figure 3). The main functions are: 
- manual, automatic and mass import of time series data (including e.g. automatic adding of new values of ongoing measurements and automatic creation of station and time series metadata during mass import of time series data)

- gap detection, quality and format consistency checks and recording of detailed metadata during import and storage of time series data in the database

- spatial analysis and search functions (e.g. distance to next station with same measured parameter), calculation of indicators (e.g. trend) and visualization of time series data and gaps

- gap filling toolbox with rule based selection of interpolation methods (e.g. inverse distance weighting with elevation correction, linear regression, nearest neighbor) and recording of detailed information on used methods and time series data for each gap

- export of data in original temporal resolution or aggregated export to specific time steps including maximum and minimum values

- API for direct data access from external tools.

Simulated and measured time series data usually have a spatial reference, e.g. a point that they have been measured at or a representative area. These virtual or real measurement stations are stored as separate datasets with name, coordinates, elevation and more information (e.g. year of establishment and closing, picture of the station). Regarding to the given spatial position and type of station the station will be automatically linked and displayed in a map (see figure 4).

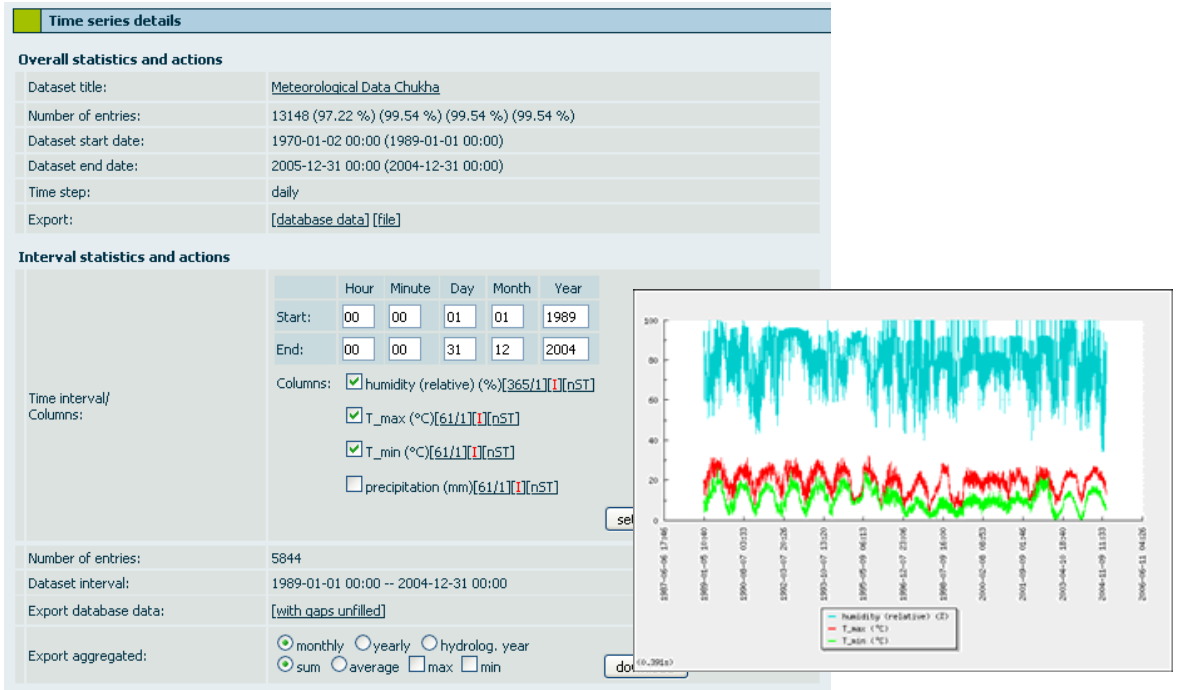

Fig. 3. Data overview of stored time series data in RBIS

\subsection{Geodate Management and Visualization (RBISmap)}

In order to manage and visualize spatial data the module RBISmap was developed. RBISmap also uses open source software like the UMN MapServer to create maps and OpenLayers with Ajax techniques for a user friendly display of map data in a web browser. RBISmap can handle vector data (ESRI Shapefile) and raster data 
(GeoTIFF, JPEG, ...). Each uploaded dataset has to be described by metadata following the ISO 19115 standard for geographic information metadata before the data can be used elsewhere (e.g. as a layer in maps). The user is demanded to fill in all information required according to the standard that cannot be automatically extracted from the dataset itself. The newly created metadata dataset is automatically linked to all maps, in which the layer is used. The linkage allows an easy switching between metadata and associated maps.

Maps are visualized in a user friendly, feature-rich frontend, including zooming/moving the map, changing the order of the displayed layers, searching for geometry objects and displaying their attribute values (figure 4). Stations and all datasets with given coordinates (e.g. soil, vegetation) can be automatically linked to a map and back which opens the possibility to search for datasets not only by metadata but also by their spatial location in a map.

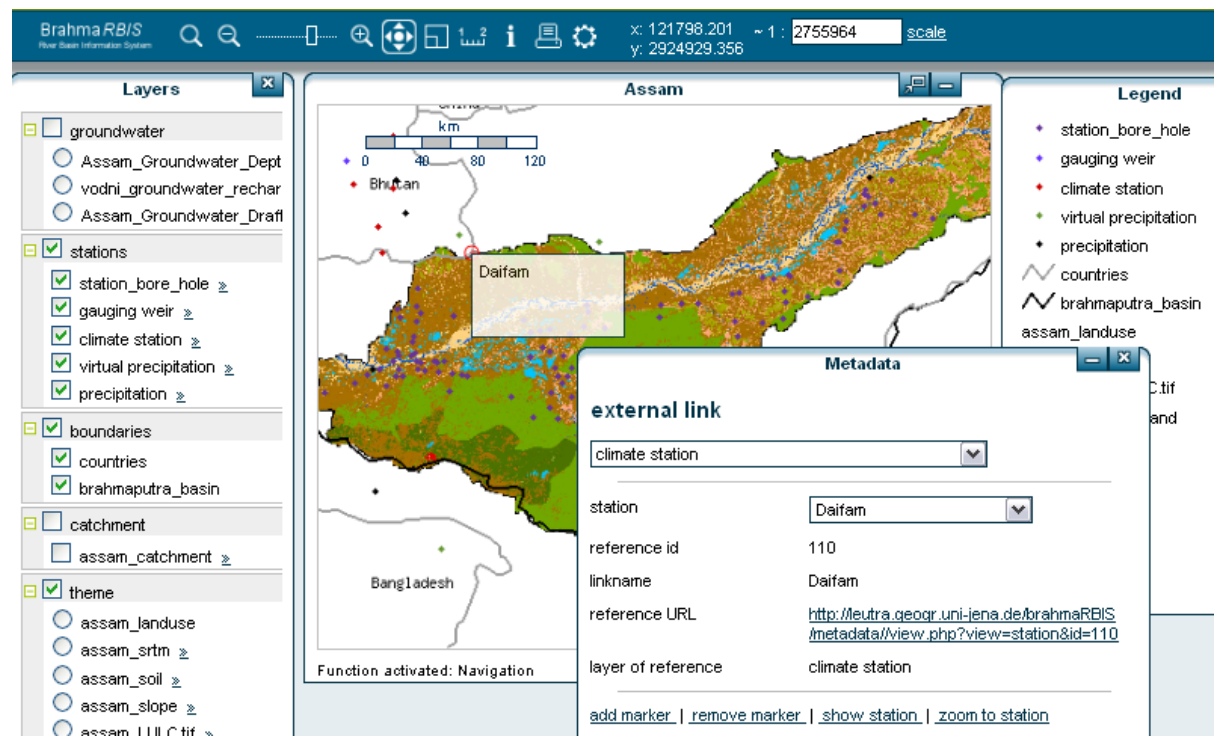

Fig. 4. Visualization of geodata and linkage between station and station metadata

\subsection{Further RBIS Modules}

In order to meet the rising demands in multidisciplinary research projects RBIS is continually extended by additional modules which can be easily added or modified as described in section 3.1. An important one is RBISdoc which allows adding an unlimited number of files (e.g. documents, images) to other RBIS datasets independent from the described data. RBISdoc furthermore offers a possibility to describe uploaded or linked documents (e.g. project proposal, master theses, paper, web links). The module RBISobserv has been developed to describe field observations (e.g. field trip, site inspection, water and soil sample) by a short description together with their spatial relation and related documents or files (e.g. pictures). Another module (RBISsoil) was developed to store soil profile and horizon 
data with a special focus is on hydrologically important parameters. Vegetation information (e.g. canopy height or leaf area index) can be stored in the RBIS module RBISbio.

For the description of future development scenarios and the management of related environmental indicators along with their values the module RBISind provides various functionalities. Indicator values (e.g. single value, time series data, diagrams, maps) can be associated to scenarios and optional to regions (e.g. hydrological catchment). New RBIS modules are under development to cover additional data types emerging from ongoing research projects.

\section{RBIS Role in the ILMS Workflow}

RBIS is a standalone application, but can also be used within the integrated ILMS workflow. As shown in the previous sections RBIS is capable of storing different types of data. In order to maximize interoperability, each ILMS component features RBIS data $\mathrm{I} / \mathrm{O}$ interfaces that allow the extraction of input data from RBIS and the storage of result data to RBIS.

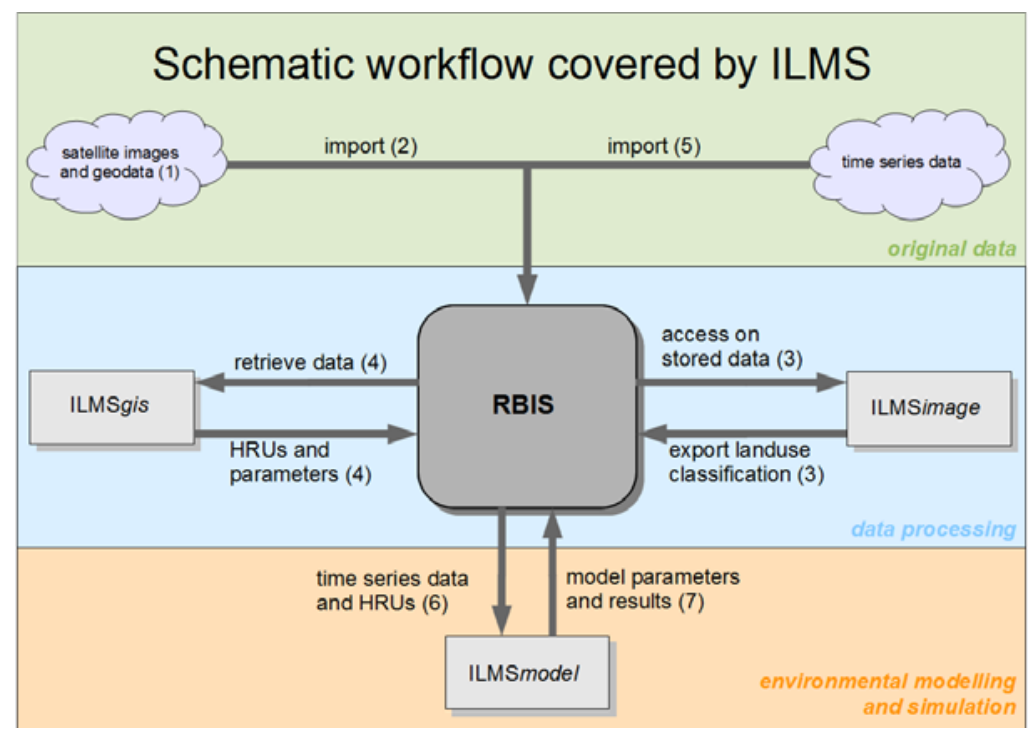

Fig. 5. Schematic workflow covered by ILMS showing the role of RBIS and the data flow between different ILMS components

Figure 5 sketches the following example workflow, using RBIS as a central data store and providing flexible and easy data exchange between ILMS components:

1. Collection of satellite images and available geodata of a defined region, including re-projection of data to a defined Spatial Reference System.

2. Import of the data into RBIS together with detailed meta-information. 
3. Direct access of stored satellite images by ILMSimage and export of a produced land use classification to RBIS.

4. Import of RBIS data to ILMSgis (i.e. DEM, land use classification, soil and geology data) and export of derived HRUs and parameter files.

5. Collection of measured time series data and import to RBIS. Preprocessing of data by filling gaps in RBIS.

6. Export of stored time series data and HRUs to ILMSmodel (for further information see [1]).

7. Model application and storage of model results in RBIS.

8. Preparations of final results and upload to RBIS for presentation.

As described in the above workflow RBIS is able to support the complete data flow starting at the original data from several sources via different processing steps to intermediate and final results consisting of simulated time series data or generated maps as base for subsequent decision making processes. The maintenance of metadata at each step also ensures transparency of data origin, change and processing as well as the reproducibility of results.

\section{Use of RBIS in Research Projects}

RBIS is used in many national and international research projects with different combinations of RBIS modules. Most of the RBIS instances are hosted at the Department of Geoinformatics, Hydrology and Modelling at the Friedrich Schiller University of Jena, but a few also in other institutions like the Institute for Technology and Resources Management in the Tropics and Subtropics at the Cologne University of Applied Sciences. Examples are the BrahmaRBIS created during the EU-Project BRAHMATWINN (twinning European and South Asian river basins to enhance capacity and implement adaptive integrated water resources management approaches - www.brahmatwinn.uni-jena.de) and instances created during other national funded projects, e.g. the BMBF projects ILMS (Integrated Landscape Management System - http://ilms.uni-jena.de), DINARIO (Climate change, landscape dynamics, land use and natural resources in the Atlantic Forest of Rio de Janeiro http://dinario.fh-koeln.de) and LUCCI (Land Use and Climate Change interactions in the Vu Gia Thu Bon River Basin/Central Vietnam - http://www.lucci-vietnam.info).

\section{Summary and Outlook}

Due to its modular structure and interfaces RBIS is a flexible environmental information system that can be used in a wide range of applications related to integrated landscape management. On the one hand RBIS is designed as standalone modular application to provide support for sophisticated data management and data sharing in multidisciplinary research projects independent of tools used in the different disciplines. On the other hand the example of ILMS shows its ability to work as a central data store by providing flexible data exchange functionalities and services, allowing related tools and application to easily access the stored data and metadata. 
Further work will focus on the development of additional RBIS modules and the implementation of various OGC standards like Catalog Web Service (CSW) or Sensor Observation Service (SOS) to provide standardized interfaces for data access and exchange that further enhance the RBIS flexibility and open it as a central data management system to an even broader range of applications.

\section{Acknowledgments}

We would like to thank the German Federal Ministry of Education and Research for funding the development of ILMS and RBIS in the course of the program "Entrepreneurial Regions - the BMBF Innovation Initiative for the New German Länder'.

\section{References}

1. Kralisch, S., Zander, F., Krause, P.: Coupling the RBIS Environmental Information System and the JAMS Modelling Framework. In: Anderssen, R., Braddock, R., Newham, L. (eds.) Proceedings of the 18th World IMACS Congress and MODSIM 2009 International Congress on Modelling and Simulation, Cairns, Australia, pp. 902-908 (2009)

2. Kralisch, S., Krause, P.: JAMS - A Framework for Natural Resource Model Development and Application. In: Voinov, A., Jakeman, A., Rizzoli, A. (eds.) Proceedings of the iEMSs Third Biannual Meeting "Summit on Environmental Modelling and Software", Burlington, USA (2006)

3. Matejka, E., Reinhold, M., Selsam, P.: IMALYS - an automated and database-integrated object-oriented classification system. In: GEOBIA 2008 - Pixels, Objects, Intelligence: Geographic Object Based Image Analysis for the 21st Century, Proceedings GEOBIA 2008, Calgary, Kanada (2008)

4. Schwartze, C.: Deriving Hydrological Response Units (HRUs) using a Web Processing Service implementation based on GRASS GIS. In: Geoinformatics FCE CTU 2008, Workshop Proceedings, Prag, vol. 3 (2008) 